\title{
Using magnetic material to repulse electrons in Axxent eBT for skin preservation during early-stage breast cancer conservative intra-operative radiotherapy
}

\author{
Li Wang, PhD', Prof. Jia-Ming Wu, PhD 2,3 \\ 'Department of Radiation Oncology, Yunnan Tumor Hospital, the Third Affiliated Hospital of Kunming Medical University, Kunming, Yunnan, \\ China, ${ }^{2}$ Department of Heavy lon Center of Wuwei Cancer Hospital, Gansu Wuwei Academy of Medical Sciences; Gansu Wuwei Tumor \\ Hospital, Wuwei City, China, ${ }^{3}$ Department of Medical Physics, Chengde Medical University. Chengde City. Hebei Province, China
}

\begin{abstract}
A miniature electronic X-ray source was employed in this study to deliver intra-operative radiotherapy (IORT) within breast lesions. A flexible magnetic material was used to reduce breast skin radiation damage. Total prescribed dose was $20 \mathrm{~Gy}$ at balloon surface, with breast tumor bed wrapped around balloon applicator. A flexible magnetic material, called 'neodymium-iron boron' (NdFeB) + alloy-49 was applied to cover lesion's surface to preserve the skin and to reduce electron contamination raised from photon-induced low-energy electrons, with tissue less than $1 \mathrm{~cm}$ between applicator surface and breast skin. The reduction of electron contamination using NdFeB + alloy- 49 with an applicator-skin distance of $20 \mathrm{~mm}$, ranged from $7 \%$ to 10\%, while with an applicator-skin distance of $10 \mathrm{~mm}$ for balloon volumes from 30 to $50 \mathrm{~cm}^{3}$, it ranged from $4 \%$ to $6 \%$ only. $\mathrm{NdFeB}+$ alloy- 49 magnetic material was efficient in repulsing photon-induced low-energy electrons for skin preservation to compensate for deficiency of tissue over less than $1 \mathrm{~cm}$ gap between the surface of applicator and the breast skin.
\end{abstract}

J Contemp Brachytherapy 2021; 13, 5: 575-582 DOI: https://doi.org/10.5114/jcb.2021.109933

Key words: neodymium-iron boron magnet, electronic brachytherapy, early-stage breast cancer, IORT, skin dose, skin sparing, alloy-49.

\section{Purpose}

A 5-year ipsilateral breast tumor recurrence (IBTR) in targeted intraoperative radiotherapy (TARGIT) arm was $3.3 \%$ in intra-operative radiotherapy (IORT) arm and $1.3 \%$ in whole breast irradiation (WBI) arm $(p=0.042)$. Overall recurrence risk was also higher in IORT arm, with hazard ratio $(\mathrm{HR})=1.44$ and $p=0.053$ [1]. At our institution, Axxent eBT system (Xoft) are used to treat early-stage breast cancer. An isotope-free miniature $\mathrm{X}$-ray source is used to generate a $50 \mathrm{KV}$ photon spectrum to deliver intra-operative radiotherapy (IORT) [2-4]. Patient is placed under general anesthesia to receive IORT immediately after breast-conserving surgery. An appropriately sized Axxent balloon applicator is implanted into the patient, based on its' tumor cavity size and shape. A computed tomography image of the implanted balloon is used to develop eBT brachytherapy treatment plans. A single-dose of 20 Gy provided by Xoft X-ray radiation is delivered for patient's treatment using a generator at the balloon sur- face [5]. Once IORT is delivered, the single-use disposable applicator is removed, and surgery can be completed.

Volume needed for treatment is calculated based on target area with a $10 \mathrm{~mm}$ thickness of the soft tissue surrounding different balloon sizes. Interface of the balloon with a wound bottom is appropriately sutured to avoid displacement and bursting during electronic brachytherapy dose delivery. The skin must be retracted from the applicator to minimize the dose delivered to the skin surface. At the same time, unacceptable delivery occurs if minimum distance from balloon surface to skin surface is less than $7 \mathrm{~mm}$ in early-stage breast cancer IORT [6]. The sterilized applicator is inserted by a surgeon and radiation oncologist in a correct position, with an entrance angle to direct electronic source through underlying tissues to the target. Surgeons and radiation oncologists must ensure that at least $1 \mathrm{~cm}$ of all breast tissue in the cavity covers the applicator, so that no part of the skin is positioned less than $1 \mathrm{~cm}$ away from the applicator. Because Asian female breasts tend to be thin and 
small, the dissected skin surface usually cannot meet the requirement of a $1 \mathrm{~cm}$ distance. The current study was performed in a phantom to simulate a situation, in which patient's skin distance was less than $1 \mathrm{~cm}$. A flexible magnetic material called 'neodymium-iron boron' (NdFeB + alloy-49) [7] was used to reduce breast skin radiation damage, especially when the gap between applicator surface and patient's skin was less than $1 \mathrm{~cm}$.

Breast-conserving therapy cannot benefit women living in developed countries and their remote areas because of travel distances between their homes and radiotherapy centers. In addition, they cannot stay or travel daily over such distances for the duration of one and half months of post-operative radiotherapy. As a result, they must choose mastectomy. These women could undergo breast-conserving therapy in one session with this novel approach.

\section{Material and methods}

A flexible water-cooling Xoft Axxent source (Xoft Inc., USA) catheter with a $25 \mathrm{~mm}$ length and $5.4 \mathrm{~mm}$ diameter was positioned inside an applicator for early-stage breast cancer treatment in this study. We adopted the AAPM TG43 report [8-11] for dose calculation and implemented this approach to all dosimetry parameters in BrachyVision $^{\mathrm{TM}}$ treatment planning system (TPS) for Xoft Axxent source. A dose of 20 Gray (Gy) X-ray radiation was delivered from a generator to the balloon surface to a small very high-dose region close to the applicator, which attenuates quickly $\left(\frac{1}{r^{3}}\right)$ from 20 Gy on the balloon surface to about $5 \mathrm{~Gy}$ at $1 \mathrm{~cm}$ from the balloon surface [12]. Xoft has three energy modes of 40,45 , and $50 \mathrm{KVp}$, and we double-checked our own office standard only for a well chamber calibration coefficient of $50 \mathrm{KVp}$; therefore, $50 \mathrm{KVp}$ was used for this study only and in clinical practice.

This experimental design was motivated by small and thin breast sizes of Asian female patients, in which the skin surface usually cannot meet the $1 \mathrm{~cm}$ requirement during surgery. Therefore, a magnetic material of $\mathrm{NdFeB}$ + alloy-49 was used to repel photon-induced low-energy electrons for skin preservation to compensate for deficiency of tissue thickness, resulting in a gap of less than $1 \mathrm{~cm}$ between the applicator surface and the breast skin.

\section{IORT technique and procedure - assessing balloon size}

Since the patient's skin dose is related to the balloon size, and the entire breast tissue in the cavity opposes the applicator at a distance of no less than $1 \mathrm{~cm}$ during IORT treatment, once our experiment's results proved to be useful, the diameter of cavity had to be evaluated with a tool after tumor removal. The Xoft balloon shaft has a series of small ports on the distal and proximal sides of the balloon to remove small pockets of seroma or blood, which might accumulate around the balloon. Some factors may influence the study results theoretically when conducted in clinical practice, such as seroma, blood, and cavity distortion. To avoid a significant amount of blood oozing from capillaries during radiotherapy, hemosta- sis of the breast wound must be rechecked. A distortion could potentially occur owing to cavity formed around the applicator. At the same time, one must note that the dose delivered to target tissues can be changed due to the distortion caused by the cavity. The usual size of the applicator was 30,40 , or $50 \mathrm{~cm}^{3}$. A purse-string suture was used to conform the tissue and skin over the balloon in lumpectomy cavity during treatment. The dose to target tissues depended on how well the purse-string stitch was made. To avoid the skin being positioned less than $1 \mathrm{~cm}$ from the applicator, the stitch had to be made not within subcutaneous tissues, but deep into the whole cavity edges through the breast tissue. A major requirement should be followed when the balloon applicator is in place, so that the entire breast tissue in the cavity opposes the applicator at a distance of no less than $1 \mathrm{~cm}$ between the skin and the applicator [13].

\section{Neodymium-iron boron (NdFeB) magnetic deflector and alloy-49 magnetism saturation material}

For neodymium-iron boron (NdFeB), a permanent magnet of alloy-49 deflector with dimensions of $15 \mathrm{~cm}$ $\times 15 \mathrm{~cm} \times 0.1 \mathrm{~cm}$ was used as a photon-induced electron contamination deflector. The weight was approximately $78.5 \mathrm{~g}$ for each of magnetic deflectors, with a $100 \mu \mathrm{m}$ thick $\mathrm{NdFeB}$ alloy-49 coating on $1 \mathrm{~mm}$ thickness industrial flexible rubber. The surface with coated magnetic material $(\mathrm{NdFeB})$, always faces the patient when used.

A magnetic saturation material called 'alloy-49' was applied, with a permeability of 1.05 to shift the magnetism at a distance of approximately $1 \mathrm{~mm}$. NdFeB permanent alloy- 49 magnet is a magnet with robust performance within contemporary magnets. Its main raw materials include metal ruthenium, pure iron, and boron-iron alloy. Alloy-49 conforms to spec ASTM A753 alloy type 2, MIL-N-14411 composition interface 3 . This magnetic saturation material can shorten the magnetism at the interface of magnetic material and the skin. A Daley electronics Tesla meter (T-22A) was applied to measure strengths of magnetic field. The magnetic strength of neodymium-iron boron magnets + alloy- 49 was $0.03-\mathrm{T}$ each, which was approximately 100 of 3.0-T MRI, which was $10^{5}$ times greater than the Earth's magnetic field. Magnetic influence was evaluated, when NdFeB alloy-49 was near other common electronic devices, such as computer monitors and kilovolt generators [14].

\section{Dosimetry of Xoft}

Calibrations were performed by comparing well-type ionization chamber response to air kerma with a NIST primary X-ray standard, and dosimetry of Axxent low-energy X-rays was commissioned [15-17]. The dosimetry of superficial therapy and the dosimetry at the surface of a phantom were recommended [18].

Axxent system incorporates a commercially available well chamber and electrometer (Models HDR 1000 Plus and MAX-4000, respectively) supplied by Standard Imaging Inc. A custom insert, with higher attenuation than Standard Imaging Inc. also provided a typical insert, spe- 
cifically for the Axxent system (Standard Imaging Inc. part number 70088).

\section{Output calibration}

The NIST (National Institute of Standards and Technology) and the University of Wisconsin ADCL (Accredited Dosimetry Calibration Laboratory) protocols were adopted for EB sources air-kerma calibration. Air-kerma strength was measured at $50 \mathrm{~cm}$ from the source surface, perpendicularly to the long axis. The detector positioning accuracy and the energy dependence of any solid water material were the main uncertainties in this study. TG-43 formalism was adopted to calculate absorbed dose to water. The absorbed dose of a reference point $1 \mathrm{~cm}$ from the source was specified using TG-43 report.

\section{A calibration curve with GAF chromic film}

GAF chromic film presented tissue equivalence $\left(Z_{\text {eff }}=6.98\right)$, with a faster polymerization (image-forming) process (readout possible after 2-4 hours) of absorption peaks, which represented energy independent of the radiation. Increased sensitivity (useful dose range of approx. 0.02-0.8 Gy) and enhanced homogeneity $(<2 \%$ for exposed film) were suggested to be used for quality assurance and dosimetry in modern radiotherapy techniques.

An international protocol was adopted for film process and optical signal analysis of the low-energy radiation distribution [19]. GAF chromic EBT2 film (ISP Technology Inc., Wayne, NJ, USA; Lot \# F05090901, expiry date April 2019) was used. Density irregularities, caused by uneven thickness of active layer by irradiating the films with a double irradiation technique were removed, which effectively reduced non-uniformity of GAF chromic film. A double exposure technique was adopted to deliver an initial dose of 2 Gy to each film on a linear accelerator before irradiation, and was applied to the film to achieve $2 \%$ of variation in dose uniformity [20]. Films were fabricated as $50 \mathrm{~mm} \times 50 \mathrm{~mm}$ squares for advanced exposure prior to the study as a double exposure technique, and then were embedded into a solid water phantom at a depth of $5 \mathrm{~cm}$. Because of layer separation extending up to $8 \mathrm{~mm}$, a frame of 40 pixels (approx. $1 \mathrm{~cm}$ ) from film edges was excluded from data collection. An $11 \mathrm{~cm} \times 11 \mathrm{~cm}$ field size at SAD (source-to-axis distance) of $100 \mathrm{~cm}$ was conducted for radiation delivery with a photon energy of $6 \mathrm{MV}$ of Elekta linear accelerator. A graded dose from $0.1 \mathrm{~Gy}$ to $0.2 \mathrm{~Gy}$ in a step size of $0.1 \mathrm{~Gy}$ was applied for calibration curve measurements.

\section{Phantom measurements of photon-induced electrons repulsed by NdFeB + alloy-49 in a linear accelerator}

The magnetic deflection power was assessed with Elekta Synergy 6-MV photon beam at $11 \mathrm{~cm} \times 11 \mathrm{~cm}$, by placing the chambers or GAF chromic film in a solid water phantom, as shown in Figure 1.

A PTW Markus parallel plate ionization chamber in a solid water stack phantom with a thickness of $1.4 \mathrm{~cm}$ was used to measure magnetic repulsion of the photon-

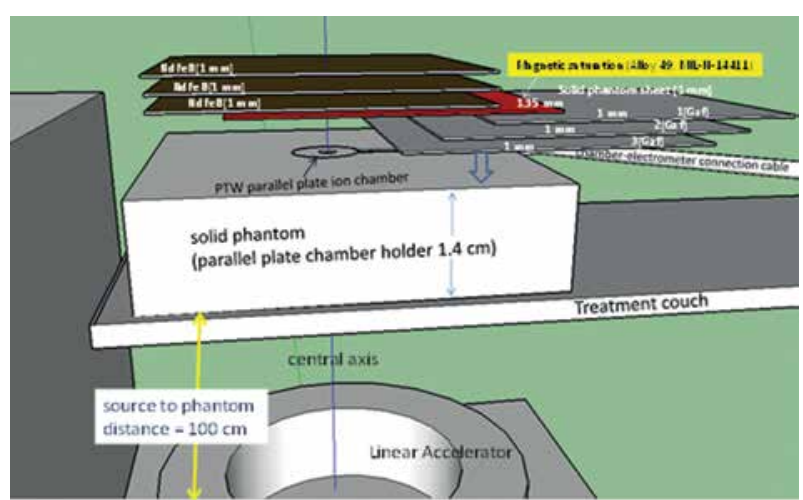

Fig. 1. Experimental setup of photon-induced electron repulsion phantom measurements using $\mathrm{NdFeB}+$ alloy- 49 under 6-MV high-energy photon beam linear accelerator. The $\mathrm{NdFeB}$ side was facing parallel plate ionization chamber for exit dose electron contamination repulsion measurement. Conversely, the rubber side of magnetic material facing plate ionization chamber has no function in reducing electron contamination

induced electron contamination in a high-energy medical linear accelerator [21]. A PTW Unidose model 30010 electrometer at $300 \mathrm{~V}$ of bias voltage was connected through a triaxial cable to chambers. The influence of various $\mathrm{NdFeB}$ layers + alloy-49 in various solid phantom sheets was measured to obtain exit dose build-up curves on the central axis for open field to estimate optimal electron contamination repulsion. The magnetic electron deflector was made with a thickness of $1 \mathrm{~mm}$ of $\mathrm{NdFeB}$ attached by a $1 \mathrm{~mm}$ thick rubber. $\mathrm{NdFeB}$ side faced a parallel plate ionization chamber for electron contamination repulsion measurement, while facing the rubber side to the parallel plate ionization chamber did not reduce electronic contamination. A magnetic saturation material alloy- 49 was placed in front of $\mathrm{NdFeB}$ to shift the magnetism on the interface of magnetic material and the skin, as shown in Figure 1. Electron contamination repulsion rate was defined by a ratio of reading with $\mathrm{NdFeB}$ side + alloy- 49 facing parallel plate ionization chamber to that of a p-p chamber reading without any magnetic material. All the measurements were repeated 5 times to ensure that the deviation of electrometer readings gathered from the ion chamber was less than $0.5 \%$. All the data were calculated or measured for population standard deviation and sample standard deviation. The overall population standard deviation was $0.048 \%$, and the sample standard deviation was $0.051 \%$. The standard errors of mean and $95 \%$ confidence interval were $0.016 \%$ and $0.031 \%$, respectively.

GAF chromic film was placed at positions 1, 2, and 3 (as shown in Figure 1), or with more thickness in a solid water phantom to compare the exit dose curves acquired by a parallel plate chamber to examine electron contamination repulsion ability.

\section{Film processing}

Epson Expression 10000XL scanner was used for the film scanning in 48-bit RGB mode, and the data were saved in a tagged image file format (TIFF). To obtain an 
H-D calibration curve, the optic density to dose conversion was analyzed using VariSoft for imaging processing. To reduce the variation in OD to within $2 \%$, the films were scanned in a landscape orientation [22]. To reduce temperature-dependent effects, the experiments and film processing were conducted at room temperature [23]. All optical density conversions were performed within one day after irradiation to avoid time effects during the measurements. Forceps were used to manage GAF chromic film to minimize scratching and fingerprints. OD, net optical density was determined using the following equation:

$$
\mathrm{OD}=\log _{10}\left(\mathrm{I}_{0} / \mathrm{I}\right),
$$

where $\mathrm{I}_{0}$ was the background (i.e., the scanner signal for an unexposed film), and I was the scanner signal for the exposed film.

\section{Red filter}

The net optical density resulted in an H-D curve as a function of radiation exposure or dose. To increase the gradient of the H-D curve, a red filter was placed on GAF films to increase the resolution of dose-OD response curves [24] during film scanning. By subtracting the reading for base signal from the measured optical density and to obtain the net optical density, the quantity of radiation dose was derived.

\section{Photon-induced electrons repulsed by NdFeB + alloy-49 in Xoft}

Three sets of different cavity volumes of 30,40 , and $50 \mathrm{~cm}^{3}$ solid water phantoms were made to simulate the balloons inside breast-conserving surgery cavities, and to measure the repulsion of photon-induced electrons by $\mathrm{NdFeB}+$ alloy-49 in Xoft (Figure 2).

The balloon was placed at three different orientations of catheters to the surface angle for surface dose mea-

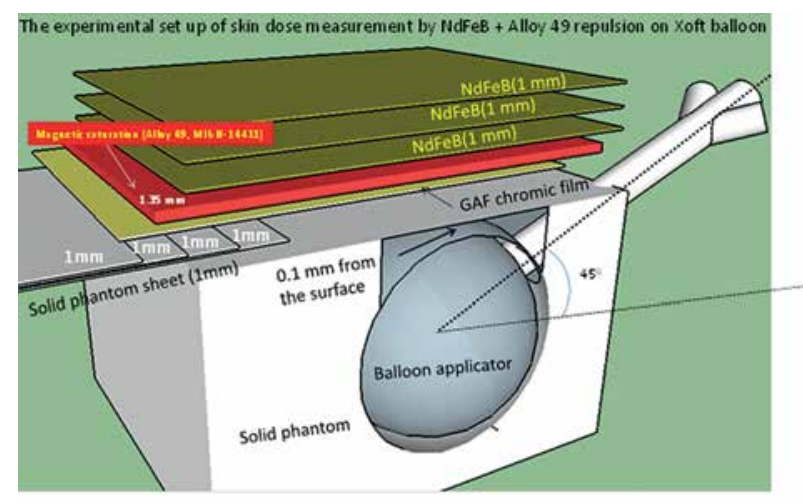

A set of Padio-dromatic film i RCFi were placed adjacent to the skin surface and fix in place with out movement with tapes in order to measure the skin dose.

Fig. 2. A sheet of GAF chromatic film was placed adjacently to the phantom surface and kept in place with transparent tape on the breast phantom, with the catheter at an angle of $45^{\circ}$ oblique to the surface, to measure the skin dose in a single-dose IORT. A set of radio-chromatic film (RCF) were placed adjacent to the skin surface and fix in place without movement with tapes in order to measure the skin dose surement. The constructed design of the balloon cavity surface-to-phantom surface distance was $0.1 \mathrm{~mm}$, and a phantom skin-like bridge could be created by adding the solid water phantom, $1 \mathrm{~mm}$ by $1 \mathrm{~mm}$, to any desired thickness. Skin dose measurement was performed with a stationary dwell position located at the balloon isocenter, providing the predicted dose of $5 \mathrm{~Gy}$ at $1 \mathrm{~cm}$ from the balloon surface. The prescribed dose was assigned to the skin surface if the distance between the balloon surface and the skin surface was less than $10 \mathrm{~mm}$. Measurements were also performed when the minimum distance from the balloon surface to the skin surface was less than $10 \mathrm{~mm}$. Considering the patient load tolerance of magnetic materials and the efficiency of repulsion of electronic contamination, only three layers of $\mathrm{NdFeB}+$ alloy- 49 sheets were placed on the phantom surface to simulate clinical situation. GAF chromic film was then embedded between the NdFeB sheet bottom and the top of solid water phantom layers for the skin dose measurement. The repulsion rate of electron contamination was defined by the ratio of dose converted by a GAF film on the phantom surface-air interface to that of $\mathrm{NdFeB}$ side-facing GAF film.

\section{Balloon insertion orientations to optimize skin surface dose delivery}

According to the operation procedure, the balloon insertion orientations to optimize skin surface dose delivery were also evaluated. Small distance between the balloon and the skin surface could result in a skin dose escalation. The symptom of telangiectasia is believed to be associated with late skin toxicity if the distance from the balloon to the skin is short. Anisotropy, with constriction of isodose distribution at the proximal end of the catheter is beneficial for preventing skin damage caused by electronic brachytherapy using balloon applicators. Such anisotropy can be considered an advantage in reducing skin dose, when the distance between the cavity and the skin is small. In this study, an optimization of the skin surface dose was simulated with various balloon-insertion orientations using home-made breast phantoms. Three different catheters, perpendicular to the surface, oblique to the surface at an angle of $45^{\circ}$, and parallel to the surface, were placed on surface orientations for the geometry-driven dose optimization analysis.

\section{Film exposure orientation by Axxent Xoft}

Radiation doses of 2 Gy were applied to GAF film in advance using an Elekta linear accelerator as previously described for dose uniformity in calibration curve measurements. $11 \mathrm{~cm} \times 11 \mathrm{~cm}$ square films were then embedded between the $\mathrm{NdFeB}$ sheet bottom and the top of polystyrene layers to simulate a clinical setup for skin dose measurement. The catheter was placed at three different surface orientations, including perpendicular to the surface, oblique to the surface at $45^{\circ}$, and parallel to the surface. Then, Nucletron Plato treatment planning system (Nucletron, Columbia, MD, USA) was used for three-dimensional dose distributions. The dose was optimized to target volume with different thicknesses of polystyrenes 
on the balloon surface by selecting multiple dwell positions at three different catheter-surface angles. A volume from the balloon surface to a $1.0 \mathrm{~cm}$ distance from the phantom surface was defined as the target volume. The results of film exposure with $\mathrm{NdFeB}$ were compared with those of film exposure without magnetic materials to evaluate the reduction in skin dose.

\section{Results}

\section{Photon-induced electrons repulsion with NdFeB + alloy-49 using linear accelerator}

The skin exit dose reductions achievable with the introduction of magnetic deflector under a 6-MV high-energy photon beam, measured by GAF chromic film and parallel plate ion chamber are presented in Figure 3 and Figure 4 , where a $2 \%$ within $2 \mathrm{~mm}$ of electrons repulsed at exit dose in this measurement is also shown.

\section{Film calibration curve}

The calibration curve was sensitive and therefore steeper when the film was scanned using a red color filter (Figure 5). The dose of radiation was then derived from a densitometer OD.

The results of chamber measurement were compared with films since the film was considered as a standard with higher resolution in relative dose measurements.

\section{Skin surface dose according to balloon-insertion orientations}

Varied sizes and shapes of balloon applicators resulted in different radiation dose ratios between tissues at the surface and the prescription dose. The reduction in dose delivery when using $\mathrm{NdFeB}$ with an applicator-skin distance from 1 to $10 \mathrm{~mm}$, ranged from $-2.44 \%$ to $-5.73 \%$, and $-4.71 \%$ to $-7.51 \%$ for balloon volume from 30 to $50 \mathrm{~cm}^{3}$, respectively (Table 1B). It was obvious that the magnetic repulsion was more effective when a large balloon was used at any depth between the balloon and phantom surfaces.

The comparisons of skin doses were obtained in readings with and without $\mathrm{NdFeB}+$ alloy-49 at the surface point. A measured dose of $81 \%$ was the lowest surface dose for the perpendicular orientation compared to the predicted dose at a $1 \mathrm{~cm}$ distance from the balloon surface. The parallel orientation had the highest surface dose of $135 \%$, while the oblique orientation showed intermediate results with a surface dose of $98 \%$, compared to the predicted dose of $1 \mathrm{~cm}$ from the balloon surface. Tables $1 \mathrm{~A}$ and $1 \mathrm{~B}$ show only the skin surface dose repulsion with $\mathrm{NdFeB}+$ alloy- 49 at $45^{\circ}$ balloon-insertion orientations in three different balloon sizes. To simulate a clinical situation, the measurement of skin dose at every thickness was conducted. Thicknesses showed in Tables $1 \mathrm{~A}$ and $1 \mathrm{~B}$ are the distances from the dose predicted point on the balloon surface (for example, $10 \mathrm{~mm}$ thickness means that the distance was $10 \mathrm{~mm}$ from the balloon surface). The reduction using $\mathrm{NdFeB}$ at an applicator-skin distance of $10 \mathrm{~mm}$ (10 mm thickness in Table 1B), ranged from $-5.73 \%$ to $-7.51 \%$ for balloon sizes of $30 \mathrm{~cm}^{3}$ to $50 \mathrm{~cm}^{3}$, and only

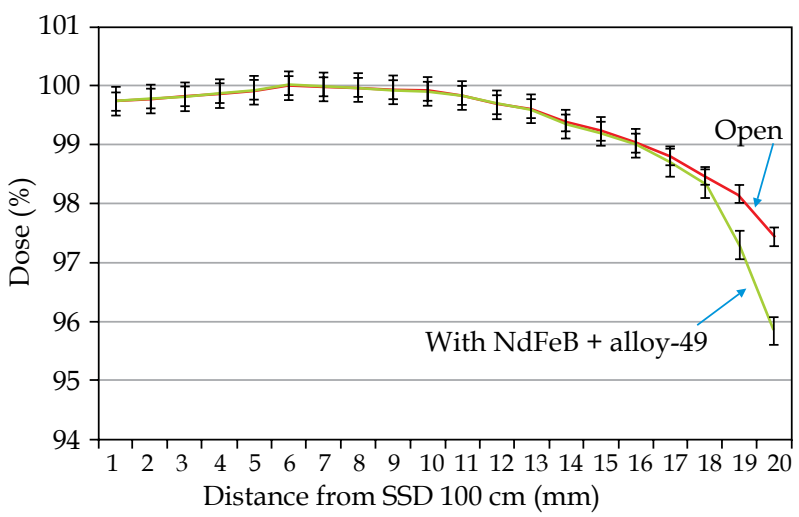

Fig. 3. Results of photon-induced electrons repulsed at exit dose measured by GAF chromic film with $\mathrm{NdFeB}$ + alloy-49 using a linear accelerator at a 6-MV high-energy photon beam

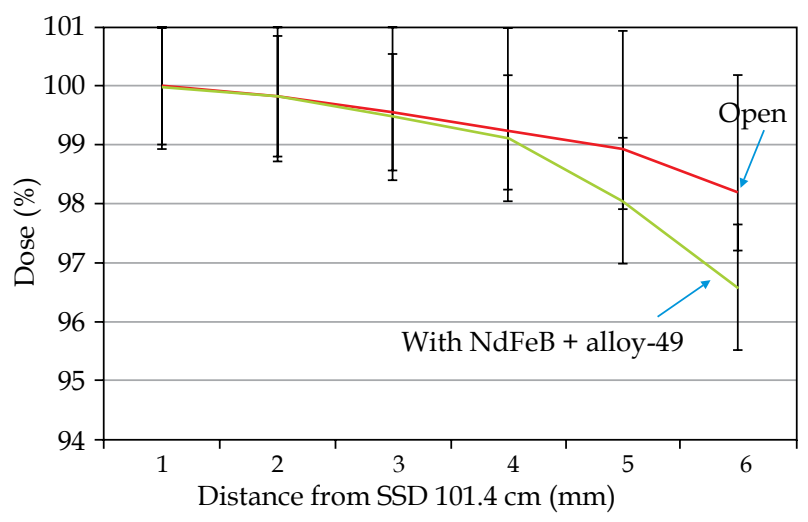

Fig. 4. Results of photon-induced electrons repulsed at exit dose measured by parallel plate ion chamber with $\mathrm{NdFeB}$ + alloy-49 using a linear accelerator at a 6-MV high-energy photon beam

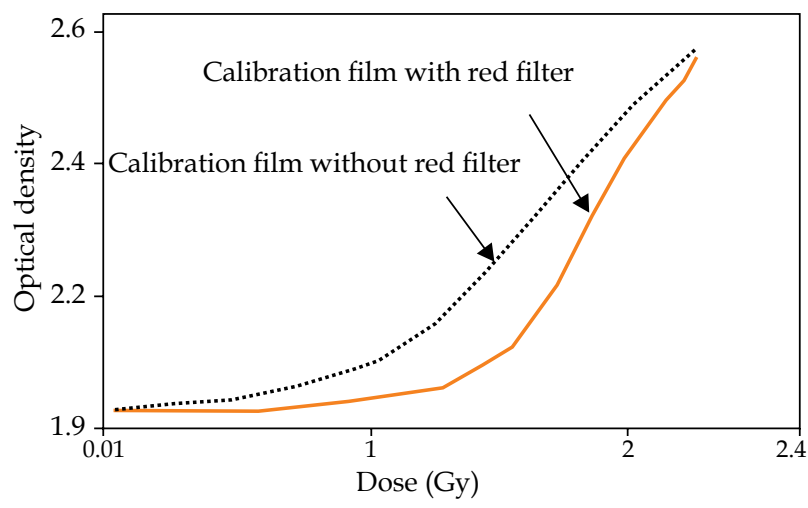

Fig. 5. Calibration curve was steeper when scanned with a red color filter, and radiation dose was then derived from the measured optical density on the film

$-1.77 \%$ to $-3.55 \%$ at an applicator-skin distance of $0 \mathrm{~mm}$ (0 mm thickness in Table 1B) for balloons with volumes from 30 to $50 \mathrm{~cm}^{3}$. The skin dose reduction was at a maximum when the distance was $10 \mathrm{~mm}$ from the $50 \mathrm{~cm}^{3}$ balloon surface. 
Table 1. Skin point dose repulsion from dose prescription of $5 \mathrm{~Gy}$ pointed $10 \mathrm{~mm}$ from the balloon surface with $\mathrm{NdFeB}+$ alloy-49 on balloon proximal point with $45^{\circ}$ balloon-insertion orientations in three different balloon sizes

\begin{tabular}{|c|c|c|c|c|}
\hline \multirow[t]{2}{*}{ Thickness $^{\mathrm{a}}(\mathrm{mm})$} & \multirow[t]{2}{*}{ Without NdFeB } & \multicolumn{3}{|c|}{ Balloon size with 3 sheets of NdFeB + alloy- 49} \\
\hline & & $30 \mathrm{~cm}^{3}$ & $40 \mathrm{~cm}^{3}$ & $50 \mathrm{~cm}^{3}$ \\
\hline 10 & $37.00 \pm 0.06 \%$ & $34.68 \pm 0.05 \%$ & $33.20 \pm 0.04 \%$ & $32.92 \pm 0.06 \%$ \\
\hline 9 & $39.10 \pm 0.05 \%$ & $36.80 \pm 0.04 \%$ & $35.37 \pm 0.06 \%$ & $35.08 \pm 0.06 \%$ \\
\hline 8 & $42.10 \pm 0.04 \%$ & $39.80 \pm 0.05 \%$ & $38.42 \pm 0.06 \%$ & $38.10 \pm 0.05 \%$ \\
\hline 7 & $45.10 \pm 0.05 \%$ & $42.80 \pm 0.06 \%$ & $41.47 \pm 0.05 \%$ & $41.13 \pm 0.05 \%$ \\
\hline 6 & $50.50 \pm 0.05 \%$ & $47.70 \pm 0.05 \%$ & $46.01 \pm 0.05 \%$ & $45.63 \pm 0.05 \%$ \\
\hline 5 & $57.10 \pm 0.05 \%$ & $54.60 \pm 0.05 \%$ & $53.30 \pm 0.06 \%$ & $52.86 \pm 0.06 \%$ \\
\hline 4 & $64.10 \pm 0.04 \%$ & $61.50 \pm 0.04 \%$ & $60.24 \pm 0.06 \%$ & $59.74 \pm 0.04 \%$ \\
\hline 3 & $70.20 \pm 0.05 \%$ & $67.40 \pm 0.05 \%$ & $66.06 \pm 0.04 \%$ & $65.52 \pm 0.05 \%$ \\
\hline 2 & $80.10 \pm 0.06 \%$ & $77.10 \pm 0.05 \%$ & $75.75 \pm 0.04 \%$ & $75.14 \pm 0.06 \%$ \\
\hline 1 & $90.10 \pm 0.05 \%$ & $86.90 \pm 0.06 \%$ & $85.55 \pm 0.05 \%$ & $84.86 \pm 0.04 \%$ \\
\hline 0 & $99.20 \pm 0.06 \%$ & $96.44 \pm 0.05 \%$ & $96.05 \pm 0.04 \%$ & $95.68 \pm 0.05 \%$ \\
\hline
\end{tabular}

aThickness as the distance from the dose prescription point at $10 \mathrm{~mm}$ from the balloon surface (for example, $10 \mathrm{~mm}$ thickness indicates that the distance is $20 \mathrm{~mm}$ from the balloon surface) with the balloon-phantom surface angle at $45^{\circ}$; The overall population standard deviation is $0.048 \%$; The sample standard deviation is $0.051 \%$; The standard error of mean and $95 \%$ confidence interval are $0.016 \%$ and $0.031 \%$, respectively

Table 2. Skin dose repulsion at less than the dose prescription at a 5 Gy point, with a certain distance from the balloon surface using NdFeB + alloy-49 on the balloon proximal point, with $45^{\circ}$ balloon-insertion orientations for balloons of three different sizes

\begin{tabular}{lcccc} 
Thickness & W $(\mathrm{mm})$ & Without NdFeB & \multicolumn{2}{c}{ Balloon size with three sheets of NdFeB + alloy-49 } \\
\cline { 3 - 5 } & & $30 \mathrm{~cm}^{3}(\mathrm{~Gy})$ & $40 \mathrm{~cm}^{3}(\mathrm{~Gy})$ & $50 \mathrm{~cm}^{3}(\mathrm{~Gy})$ \\
\hline 10 & $5 \mathrm{~Gy}^{\mathrm{b}}(4.05 \mathrm{~Gy})$ & $3.85 \pm 0.031^{\mathrm{d}}(-4.93 \%)$ & $3.81 \pm 0.03(-5.93 \%)$ & $3.77 \pm 0.033(-6.91 \%)$ \\
\hline 9 & $5 \mathrm{~Gy}(4.11 \mathrm{~Gy})$ & $3.97 \pm 0.029(-3.41 \%)$ & $3.89 \pm 0.028(-5.35 \%)$ & $3.86 \pm 0.030(-6.08 \%)$ \\
\hline 8 & $5 \mathrm{~Gy}(4.15 \mathrm{~Gy})$ & $4.02 \pm 0.023$ & $3.95 \pm 0.025$ & $3.91 \pm 0.029$ \\
\hline 7 & 5 Gy (4.18 Gy) & $4.06 \pm 0.021$ & $3.99 \pm 0.020$ & $3.97 \pm 0.024$
\end{tabular}

Thickness: the distance of the dose prescription point at a certain distance $(\mathrm{mm})$ from the balloon surface with a balloon-phantom surface angle at $45^{\circ}$; b treatment plan prescription dose: the point of dose prescription is the thickness from the balloon surface; cactual measured dose; ${ }^{d}$ Skin dose repulsion with NdFeB + alloy-49

Table 2 describes skin dose repulsion at less than predicted 5 Gy point at a certain distance from the balloon surface with $\mathrm{NdFeB}+$ alloy-45 on the balloon proximal point, with $45^{\circ}$ balloon-insertion orientations for three different balloon sizes. The reduction using $\mathrm{NdFeB}+$ alloy-49 at an applicator-skin distance of less than $10 \mathrm{~mm}$, ranged from $-4.93 \%$ to $-6.91 \%$ for balloon volumes from 30 to $50 \mathrm{~cm}^{3}$, respectively.

\section{Discussion}

Scatter electron energy induced by a $50-\mathrm{kV}$ photon beam can be compared with an interaction of high-energy photon with orbital electron. If the incident photon has very high energy that is much greater than the resting energy of electron, the photon drops most of its energy to Compton electron, therefore, the scattered photon has much lower energy. Thus, maximum scattered electron energy induced by 6-MV high-energy photon beam is approximately $0.25 \mathrm{MeV}$. The average photon energy of $50 \mathrm{KVp}$ produced by Axxent eBT used in the current study was about $17 \mathrm{KeV}$. According to Compton scattering equation, the maximum scatter electron energy from a $50-\mathrm{KeV}$ photon was $1.1 \mathrm{KeV}$ when photon scattered at an angle of $\theta=180^{\circ}$, and the maximum range in water (soft tissue) of electron energy $1.1 \mathrm{KeV}$ was $0.0055 \mathrm{~mm}$. In the present study, the electron induced by $50 \mathrm{KVp}$ photon in Axxent eBT could be neglected or repulsed by neodymium-iron boron $(\mathrm{NdFeB})+$ alloy-49. We learned that the skin dose was the sum of initial kinetic energies of all the charged ionizing particles (electrons and positrons) released by the uncharged particles (photons) in the basal and dermal layers [25].

The deflection and removal of high-energy electrons produced by a medical linear accelerator was attained by a neodymium-iron boron $(\mathrm{NdFeB})+$ alloy- 49 permanent magnetic deflector device, in which photon-induced low-energy electrons are removed by magnetism. Low-energy photon exits the skin surface and penetrates through magnetic material without contributing any backscatter dose to the skin. Therefore, removal of electron contamination from a $50-\mathrm{kV}$ electronic brachytherapy machine was achieved using $\mathrm{NdFeB}$ magnetic materials + alloy-49 magnetic saturation materials in this study.

To maintain optimal tumor cavity coverage and to minimize skin dose, the optimized Xoft Axxent balloon 
catheter and multiple dwell positions were used when the cavity-to-skin distance was small.

A lead-rubber protection sheet of $0.25 \mathrm{~mm} \mathrm{~Pb}$ equivalent is usually applied to cover the patient over the applicator in breast IORT treatments for radiation safety considerations [26, 27]. NdFeB + alloy-49 magnetic material can be used for EO sterilization only to avoid damage of volatile toxicity compounds. $\mathrm{NdFeB}+$ alloy- 49 and other parts of the devices were ethylene oxide-sterilized before use. The skin above the breast of IORT lesion was wrapped with a sterilized plastic film before applying magnetic material to the patient skin surface. Using three stacked $\mathrm{NdFeB}+$ alloy-49 layers to repulse photon-induced electrons, not only provides skin preservation but also protects the patient during radiation treatment.

This system is contraindicated for implanting a balloon applicator if the size of resected tumor cavity is not consistent with inflated volume range, and general shape of a specific balloon is selected. A patient is not considered a candidate for radiation therapy with Axxent electronic brachytherapy system, if a minimum distance from the balloon surface to the skin surface is less than $7 \mathrm{~mm}$. In certain conditions, for example, in patients with extreme or unusual anatomical features, such as extreme rib curvature or very irregularly-shaped lumpectomy cavities, the balloon applicator could become asymmetrically located, affecting conformal delivery of the radiation dose to the target tissue.

\section{Conclusions}

A magnetic material was used in a single-dose IORT to reduce breast skin radiation damage when the gap between the applicator surface and the patient's skin was either more or less than $1 \mathrm{~cm}$. The skin dose reduction using $\mathrm{NdFeB}$ at a certain applicator-skin distance could range from a minimum of $-2.44 \%$ to a maximum of $-7.51 \%$ for balloon sizes of $30 \mathrm{~cm}^{3}$ to $50 \mathrm{~cm}^{3}$. We noted that, in case of Asian patients, female breast shapes and sizes tend to be thin and small; therefore, the skin envelope usually could not meet the $1 \mathrm{~cm}$ requirement. Due to cosmesis and skin sparing required, we recommend that patients use magnetic material to repulse electrons for skin preservation during conservative early-stage breast cancer intra-operative radiotherapy.

\section{Ethical statements}

This article does not contain any studies with human participants or animals performed by any of the authors.

\section{Acknowledgments}

This work was supported by the Department of Education Foundation, Yunnan Province, China (grant No., 2020J0205).

\section{Disclosure}

The authors report no conflict of interest.

\section{References}

1. Beddar AS Biggs PJ, Chang $S$ et al. Intraoperative radiation therapy using mobile electron linear accelerators: Report of AAPM Radiation Therapy Committee Task Group No. 72. Med Phys 2006; 33: 1476-1489.

2. Shaw EG, Blackwell CR, McCullough EC et al. Matching intraoperative electron-beam fields: dosimetric and clinical considerations. Int J Radiat Oncol Biol Phys 1987; 13: 1303-1307.

3. Dinsmore M, Harte KJ, Sliski AP et al. A new miniature x-ray source for interstitial radiosurgery: device description. Med Phys 1996; 23: 45-52.

4. Beatty J, Biggs PJ, Gall K et al. A new miniature x-ray device for interstitial radiosurgery: dosimetry. Med Phys 1996; 23: 53-62.

5. Eaton DJ. Electronic brachytherapy - current status and future directions. Br J Radiol 2015; 88: 20-28.

6. Ebert MA, Carruthers B. Dosimetric characteristics of a low$\mathrm{kV}$ intra-operative $\mathrm{x}$-ray source: Implications for use in a clinical trial for treatment of low-risk breast cancer. Med Phys 2003; 30: 2424-2431.

7. Butson MJ, Wong TP, Law A et al. Magnetic repulsion of linear accelerator contaminates. Med Phys 1996; 23: 953-955.

8. Eaton DJ. Quality assurance and independent dosimetry for an intraoperative x-ray device. Med Phys 2012; 39: 6908-6920.

9. Park CC, Yom SS, Podgorsak MB et al. Electronic Brachytherapy Working Group. American Society for Therapeutic Radiology and Oncology (ASTRO) Emerging Technology Committee report on electronic brachytherapy. Int J Radiat Oncol Biol Phys 2010; 76: 963-972.

10. Rivard MJ, DeWerd L, Coursey BM. Update of AAPM Task Group No. 43 Report: A revised AAPM protocol for brachytherapy dose calculations. Med Phys 2004; 31: 633-674.

11. Rivard MJ, Davis SD, DeWerd LA et al. Calculated and measured brachytherapy dosimetry parameters in water for the Xoft Axxent X-ray source: an electronic brachytherapy source. Med Phys 2006; 33: 4020-4032.

12. Ebert MA, Carruthers B. Dosimetric characteristics of a low$\mathrm{kV}$ intra-operative $\mathrm{x}$-ray source: Implications for use in a clinical trial for treatment of low-risk breast cancer. Med Phys 2003; 30: 2424-2431.

13. Njeh CF, Saunders MW, Langton CM. Accelerated partial breast irradiation (APBI): A review of available techniques. Radiat Oncol 2010; 5: 90.

14. Damrongkijudom N, Oborn B, Butson M et al. Measurement and production of electron deflection using a sweeping magnetic device in radiotherapy. Australas Phys Eng Sci Med 2006; 29: 260-266.

15. Martignano A, Menegotti L, Veronesi A et al. Monte Carlo investigation of breast intraoperative radiation therapy with metal attenuator plates. Med Phys 2007; 34: 4578-4584.

16. Eaton DJ, Duck S. Dosimetry measurements with an intra-operative x-ray device. Phys Med Biol 2010; 55: 359-369.

17. White SA, Landry G, Fonseca GP et al. Comparison of TG-43 and TG-186 in breast irradiation using a low energy electronic brachytherapy source. Med Phys 2014; 41: 61-70.

18. Eaton DJ. Quality assurance and independent dosimetry for an intraoperative x-ray device. Med Phys 2012; 39: 6908-6920.

19. Gueli AM, Cavalli N, Vincolis RD et al. Background fog subtraction methods in Gafchromic dosimetry. Radiat Measurement 2015; 72: 44-52.

20. Lynch M, Ranade JL, Dempsey J. Characteristics of a very high sensitivity radiochromic film. Med Phys 2004; 31: 1837-1849.

21. Slobodan D, Nada T, David L. Reference radiochromic film dosimetry: Review of technical aspects. Phys Med 2016; 32: 541-556. 
22. García-Garduño OA, Lárraga-Gutiérrez JM, Rodríguez-Villafuerte $\mathrm{M}$ et al. Effect of correction methods of radiochromic EBT2 films on the accuracy of IMRT QA. Appl Radiat Isot 2016; 107: 121-126.

23. Robert J, Torbjørn E. A new information theoretic analysis of sum-of-squared-error kernel clustering. Neurol Comp 2008; 72: 23-31.

24. Weeks KJ, Litvinenko VN, Madey JMJ. The Compton backscattering process and radiotherapy. Med Phys 1997; 24: 143152.

25. Daves M, Mills D. Shielding assessment of a mobile electron accelerator for intraoperative radiotherapy. J Appl Clin Med Phys 2001; 2: 165-173.

26. 26. Krechetov AS, Goer D. Dikeman K. Shielding assessment of a mobile electron accelerator for intra-operative radiotherapy. J Appl Clin Med Phys 2010; 11: 31-51. 